\title{
Densidad poblacional del tigrillo (Leopardus pardalis) en el valle del Cerrejón, La Guajira, Colombia
}

\author{
Orlando Padilla Rivera \\ Facultad de Ciencias Básicas Universidad del Atlántico, Km 7 Ant. Vía a Puerto Colombia, Barranquilla \\ orlandopadillarivera@gmail.com \\ Erhent Madariaga \\ Helí Arciniegas \\ Facultad de Ciencias Básicas Universidad del Atlántico, Km 7 Ant. Vía a Puerto Colombia, Barranquilla \\ Bioma Consultores S.A.S, Barranquilla
}

\section{Resumen}

A pesar de su amplia distribución geográfica, los patrones de abundancia del tigrillo (Leopardus pardalis) son poco conocidos en paisajes transformados. En este trabajo se evaluó la densidad poblacional de esta especie en la Mina del Cerrejón (Guajira, Colombia) por medio de 68 días de fotocapturas con 27 estaciones de fototrampeo. Dos estimadores poblacionales fueron utilizados:1) Estimadores de probabilidad de captura y tamaño poblacional para poblaciones cerradas con datos de recaptura en el programa Capture ${ }^{\circledR}$ y 2) el índice de Lincoln-Petersen. Basados en los patrones de manchas de la piel, se identificaron ocho tigrillos: cuatro machos, dos hembras y dos individuos no sexados. La densidad de tigrillos en la zona varía entre 22 y 24 individuos $/ 100 \mathrm{~km}^{2}$, dependiendo del estimador utilizado. Las estimaciones de densidad se encuentran dentro de lo reportado en la literatura ecológica de la especie en diferentes tipos de hábitats. Por lo tanto, los resultados de este estudio sugieren que esta especie puede ajustarse a áreas intervenidas siempre y cuando haya la presencia de hábitat idóneo en el paisaje adyacente.

Palabras clave: Caribe, Fototrampeo, Fragmentación, Minería, Ocelote.

\section{Abstract}

Although the ocelot (Leopardus pardalis) has a widespread geographic distribution, there is a lack of information about density patterns of this species in transformed landscapes. In this work, the population density of this species was evaluated in the Cerrejón coal mine (La Guajira, Colombia) using 68 days of photo captures using 27 photo trapping stations. Two population estimators were used: 1 ) estimates of capture probability and population size for closed populations with recapture data in the Capture program and 2) Lincoln-Petersen index. Based on the skin patterns of the individuals, a total of 8 tigrillos were identified: four males, two females and two non-sexed individuals. According to Capture the density of ocelots in the area is 24 individuals $/ 100 \mathrm{~km}^{2}$, while the estimates with the Lincoln Petersen index were 22 individuals / 100km2. Density estimates are within the range of estimates reported for the species in previous studies in diverse types of habitats. Therefore, our results suggest that ocelots can adjust to transformed areas as long suitable habitat is present in adjacent areas.

Key words: Camera Trapping, Caribbean region, Fragmentation, Mining, Ocelot.

\section{Introducción}

Los tigrillos (Leopardus pardalis) son considerados como una especie ecológicamente importante y de carácter carismático, considerada además como especie bandera en términos de la conservación de los hábitats en los que está presente (González -Maya et al. 2013; Báez \& Trujillo 2014). Por su posición en la red trófica, tienen un rol importante al regular las poblaciones de sus presas en los ecosistemas donde habita (Eissenberg 1989; Emmons 1990; Cruz et al. 2015).

A pesar de la importancia ecológica de esta especie, la información sobre los patrones de abundancia y densidad de tigrillos en Colombia es escasa. Como evidencia existen algunas estimaciones de densidades para la Amazonia colombiana (Payan 2009), los Llanos orientales (Díaz-pulido \& Payán 2011), además de datos de abundancias en la región Caribe (GonzálezMaya et al. 2013). Sin embargo, la mayoría de los estudios sobre densidades de tigrillos provienen de áreas fuera de Colombia en paisajes relativamente conservados. Por ejemplo, en la isla Barro Colorado en Panamá se han realizado varios estudios aplicando diferentes metodologías: hábitos alimentarios con colecta de excretas (Moreno \& Giacalone 2006) monitoreo con registro de huellas (Moreno 2000; Moreno 2002; Moreno 2005), densidad con cámaras trampa (Moreno 2005),

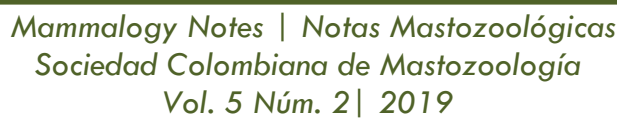


comportamiento con video filmadoras automáticas y radio telemetría manual y automática (Moreno et al. 2006 A y B; AliagaRossel et al.2006).

Debido a que $L$. pardalis ha sido registrado en diferentes tipos de ecosistemas con varios grados de intervención, es importante recolectar evidencia sobre patrones de abundancia de esta especie en paisajes intervenidos. En Colombia, L. pardalis enfrenta amenazas locales debido a la destrucción del hábitat, disminución de presas, persecución directa cuando interactúan con animales domésticos y tráfico ilegal (Rueda-Almonacid et al. 2008). A pesar de esto, no se cuentan con suficientes datos sobre los patrones de densidad de esta especie en áreas fragmentadas y más aún donde la intervención es de carácter permanente en el tiempo y de gran impacto a nivel espacial. Aunque esta especie no se encuentra en ninguna categoría de amenaza nacional (Ministerio de Ambiente, 2017) o internacional (Caso et al. 2008), la escasez de información biológica y poblacional a nivel local obstruye el diseño de planes de manejo para proteger esta especie en el medio natural.

En el presente estudio se reporta el patrón de densidad de tigrillos en el complejo minero del Cerrejón, localizado en un paisaje intervenido del Caribe colombiano. Para ello, se usaron cámaras trampa, las cuales permiten identificar individuos mediante patrones únicos de manchas (Moreno \& Bustamante, 2007; Bustamante, 2008; Noss et al. 2013). Además de proveer estimaciones de densidad en una nueva zona, esta información servirá como insumo para evaluar el monitoreo de acciones de restauración del ecosistema circundante alterado en las actividades mineras.

\section{Metodología}

Área de estudio

Este estudio se realizó en el área de la Mina del Cerrejón (ubicada entre los municipios de Albania, Barrancas y Hatonuevo en el departamento de La Guajira, Colombia ( $11^{\circ} 07^{\prime} 12.95^{\prime}$ N $72^{\circ} 35^{\prime} 09.05^{\prime}$ W) (Figura 1), zona clasificada como bosque seco tropical y matorral xerófilo tropical (Holdridge 1967) ubicada a aproximadamente a $128 \mathrm{msnm}$.

Teniendo en cuenta las coberturas asociadas y las opciones de conectividad inherentes a estas áreas, se seleccionaron tres sectores como objeto de fototrampeo: 1) las zonas restauradas o "zona de rehabilitación" con matorrales con un progreso sucesional de 20 años en su mayor parte (Báez y Trujillo 2014); 2) la vegetación de galería asociada al río Ranchería y arroyo Tabaco; 3) zonas de bosque seco conservado y conectado con las estribaciones de la serranía del Perijá en el límite de avance minero conocido como sector botadero "La Estrella" (Fig. 1).

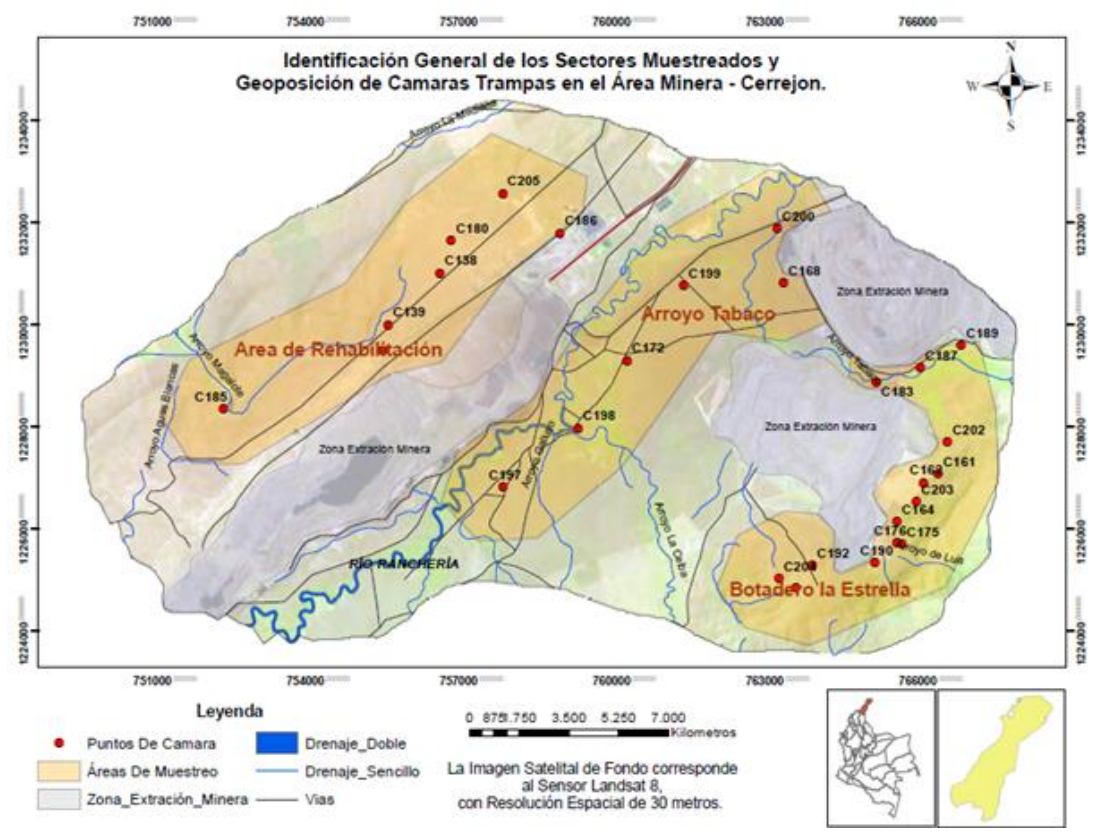

Figura 1. Área de estudio y sectores seleccionados para los muestreos. 
Métodos

El trabajo de campo se realizó durante 68 días utilizando 27 cámaras trampas, una en cada punto de registro (1836 días/cámara trampa) entre noviembre de 2013 y enero de 2014, desarrollándose en la temporada seca en la región. Las cámaras se georeferenciaron y se mantuvieron en funcionamiento las 24 horas del día durante el período de muestreo, y fueron cebadas dos veces por mes con perfumes de Feromonas "Feroz M" y "Feroz F" en Spray, ubicadas a fin de permitir el tránsito pausado de los individuos de tal manera que los animales de acercaran exponiendo ambos flancos. La restricción del estudio a un período de 68 días cumple con la exigencia del supuesto de una población cerrada necesaria para los cálculos de densidad (Otis et al. 1978; Redxtad \& Burnham 1991; Karanth \& Nichols 1998; Karanth 1995). Las estaciones fueron instaladas a distancias que variaban entre $0,5 \mathrm{~km}^{2}$ y $1,1 \mathrm{~km}^{2}$. Estas distancias fueron escogidas tomando en cuenta estimaciones de áreas vitales observadas en estudios previos en Texas (USA), Pantanal Brasileño, Amazonía peruana y el chaco boliviano, las cuales varían de 2 a 6 km² para machos y 1 a 3 km² para hembras. (Navarro 1985; Emmons 1988; Crawshaw \& Quigley 1989; Laack 1991; Moreno et al. 2012).

Para la estimación del área efectiva de muestreo, se utilizó la media máxima distancia de movimiento (mmdm). (Díaz-Pulido \& Payán 2012). Con esta medida se calculó un buffer externo alrededor de cada cámara trampa, mediante la herramienta analysis tools - proximity de Arcgis 10.1. Para calcular el área total obtenida mediante los buffers individuales, se realizó la unión de todas las áreas mediante la herramienta Analysis Tools-Overlay ArcGis 10.1. El área efectiva de muestreo correspondió a un medio (1/2) del área abarcada por estos buffers (Díaz-Pulido \& Payán 2012). Cabe anotar que parte del área de muestreo efectiva calculada para cada cámara trampa abarcó sectores de la zona de extracción minera (6\%), por lo cual fue necesario excluir dichos parches, obteniéndose así el área total de muestreo que fue $52.275 \mathrm{~km}^{2}$.

Con el objetivo de hacer el análisis del tamaño poblacional y densidad se realizaron dos aproximaciones diferentes, una utilizando el programa Capture $@($ Rexstad \& Burnham 1991) y otra con el estimador Lincoln-Petersen (Ministerio de Medio Ambiente de Colombia 2000). El programa Capture@ provee como resultado una estimación del tamaño poblacional de la especie para diferentes modelos con supuestos diferentes, estos supuestos difieren en probabilidades de captura por individuos, en el tiempo y en el espacio. Por lo tanto, el programa escoge el modelo con las mayores probabilidades de captura, errores estándar e intervalos de confianza más bajos (Otis et al. 1978; Rexstad \& Burnham 1991; Karanth, 1995; Karanth \& Nichols 1998; Díaz-Pulido \& Payán 2012). Para hallar la densidad el resultado de Capture es llevado a la siguiente fórmula:

$$
\mathrm{D}=(\mathrm{N} / \mathrm{A}) \times 100 \mathrm{~km}^{2}
$$

Dónde: D es la densidad de la población, $\mathrm{N}$ es el estimado de la población por el programa Capture, A es el área efectiva de muestreo y $100 \mathrm{~km}^{2}$ es el factor de corrección para presentar las estimaciones de densidad. El segundo estimador fue el de Lincoln - Peterson que es igualmente basado en modelos de captura y recaptura de individuos reconocibles o marcados, cuya fórmula es:

$$
\mathrm{NP}=\mathrm{MN} / \mathrm{m}
$$

Dónde: $\mathrm{M}$ es el número de animales capturados, marcados y liberados en el periodo 1; $\mathrm{N}$ es número total de animales capturados en periodo 2; $\mathrm{m}$ es número total de animales marcados y capturados el periodo 2; y, NP es el estimado del tamaño poblacional. (Ministerio del Medio Ambiente 2000). Los periodos de fotocaptura se hicieron coincidir con la transición de la época de lluvias (primer periodo) y la consolidación de la temporada de sequía en la región (segundo periodo).

\section{Resultados}

Se obtuvieron 67 fotografías correspondientes a ocho tigrillos (cuatro machos, dos hembras) y dos individuos no sexados; en un total de 1890 días/ cámara trampa, en 21 ocasiones de foto captura (Figura 2). Para el análisis estadístico los individuos fotografiados en los dos periodos fueron contados como observaciones independientes en cada periodo. En el primer periodo de muestreo (18 noviembre - 22 diciembre 2013: 34 días) se obtuvieron 32 fotografías, 8 ocasiones de capturas de tigrillos y se identificaron 4 individuos. En el segundo periodo de muestreo (23 de diciembre 2013 - 26 de enero 2014 : 34 días) se obtuvieron 35 fotografías de tigrillos, 13 ocasiones de capturas y se identificaron 4 individuos diferentes. De todos los individuos identificados, solo uno de ellos fue fotografiado durante el primer y segundo periodo de muestreo.

Mammalogy Notes | Notas Mastozoológicas

Sociedad Colombiana de Mastozoología

Vol. 5 Núm. 2| 2019 

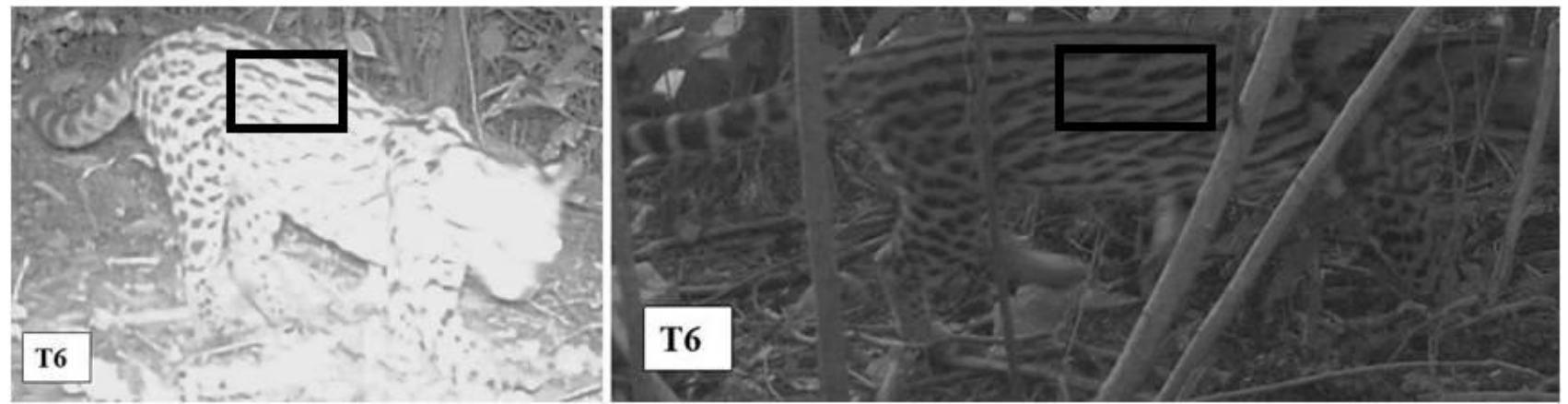

Figura 2. Imagen de individuo T6 registrado en el sector La Estrella. Los cuadros negros muestran secciones de piel usadas

para reconocer al individuo.

El promedio de las distancias máximas de movimiento de los tigrillos de acuerdo con las fotos recapturas fue de $2.28 \mathrm{~km}$ y la zona de estudio con mayor número de foto capturas fue la de arbustales y matorrales en rehabilitación con 10, seguido por el bosque seco de La Estrella con 7 y por último la zona vegetación ribereña de arroyo Tabaco con 4.

Análisis poblacional.

El modelo de variación temporal (Mt) reconoció una población con cambios específicos en el tiempo en la probabilidad de captura; bajo este supuesto el resultado del programa Capture estima la población en 13 individuos con Error Estándar \pm 1.42 en $52.75 \mathrm{~km}^{2}$; la densidad estimada es de 24 tigrillos $/ 100 \mathrm{~km}^{2}$ Error Estándar $\pm 4,5$ al 95\%. Paralelamente, también de acuerdo con el historial de foto capturas en ambos periodos con el índice de Lincoln-Petersen cuya estimación arrojó 12 individuos (Error Estándar=1,35; Desviación Estándar=1,83; Intervalo Confianza $+=14,7 ; \mathrm{IC}-=9,29$ al 95\%). a nivel de densidad y basados en el estimativo de Lincoln-Petersen se calculan 22 tigrillos $/ 100 \mathrm{~km}^{2}$ Error Estándar $\pm 2,70$; Desviación Estándar=1,83; Intervalo Confianza = 14,7 - 9,29 al 95\% de confianza.

\section{Discusión}

La distribución de L. pardalis es amplia para el complejo carbonífero del Cerrejón, registrándose en todas las áreas de estudio y haciendo uso de la vegetación abierta presente en las áreas abiertas de rehabilitación, el bosque seco en el sector de la estrella y vegetación de galería del arroyo Tabaco y río Ranchería; con acentuada presencia en los sectores intervenidos de TabacoRanchería y Rehabilitación. Los datos de densidad y abundancia presentados en la zona se consideran típicos de la especie encontrándose en el rango recopilado por Rueda-Almonacid (2008) y tomando en cuenta diez publicaciones a lo largo de su rango de distribución geográfica, sólo con el método de cámaras trampa (Trolle \& Kery 2003,2005; Dillon, 2005; Maffei et al. 2005; Di Bitetti et al. 2005; Haines et al. 2006; Moreira et al., 2007; Moreno y Bustamante 2007; Bustamante 2008; Torres- Romero et al. 2017).

Se considera que la especie L. pardalis, es naturalmente más abundante que otras especies de félidos simpátricos y que sus densidades en toda su área de distribución varían entre 5 y 100 individuos $/ 100 \mathrm{~km}^{2}$ en la mayoría de los hábitats de la región Neotropical de tierras bajas. Esta relativa tolerancia a hábitats perturbados y fragmentación permiten a esta especie sostener poblaciones viables a largo plazo (Caso et al. 2008). Además, la presencia de poblaciones de presas es un factor de alta relevancia e influencia sobre la densidad de carnívoros (Karanth \& Nichols 2002; Seidensticker \& Lumpkin 2004; Roy et al. 2015). Tanto en este estudio como en estudios anteriores (González -Maya et al. 2013, Báez \& Trujillo 2014), se ha registrado la presencia en la zona de presas como Ortalis ruficauda, Marmosa robinsoni, Dasyprocta punctata, Sylvilagus floridanus, Mazama gr. americana, Dasypus novemcinctus, Procyon cancrivorus.

Finalmente, hay que reconocer que las áreas silvestres a abaja altura, poco alteradas y adyacentes del sistema de la serranía de Perijá son un refugio de fauna incluidas las 5 de las 6 especies de félidos nativos de Colombia, lo que genera la oportunidad de que los esquemas de conservación y restauración del complejo minero fomenten la presencia de los predadores flexibles

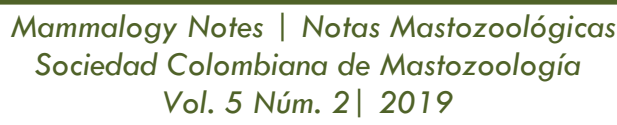


en cuanto al uso de hábitats como es el caso del tigrillo. Es importante que el trabajo de monitoreo en el área de estudio continúe revisando las tendencias de esta población en el tiempo, con el fin de que en su momento orienten el diseño de la conectividad áreas silvestres internas y corredores de dispersión hacia fuera el predio minero.

\section{Agradecimientos}

Este estudio fue realizado en medio de las actividades de monitoreo de la biodiversidad del complejo minero Cerrejón, en el contexto del estudio poblacional del tigrillo ejecutado por Bioma Consultores S.A.S en el año 2014 y en su momento supervisado por Lina Báez especialista en biodiversidad a quien agradecemos su apoyo durante la fase de campo. Agradecemos también a Andrés Felipe Suárez-Castro por su apoyo durante el ajuste de este manuscrito.

\section{Referencias}

ALIAGA-ROSSEL, E. et al. 2006. Ocelot (Leopardus pardalis) predation on agoutis (Dasyprocta punctata). Biotropica 38 (5): $691-694$. BÁEZ, L. \& TRUJILLO, F. (Editores.). 2014. Biodiversidad en Cerrejón. Carbones de Cerrejón,

Fundación Omacha, Fondo para la Acción Ambiental y la Niñez. Bogotá, Colombia. 352 p.

BUSTAMANTE, A. 2008. Densidad y uso de hábitat por los tigrillos (Leopardus pardalis), pumas (Puma concolor) y jaguares (Panthera onca) en la parte sureste del área de amortiguamiento del parque nacional corcovado, península de Osa. Costa Rica. Tesis De Maestría. Instituto Internacional En Conservación y Manejo De Vida Silvestre. 137 p. CASO, A., et al. 2008. Leopardus pardalis. The IUCN Red List of Threatened Species 2008: e. Downloaded on 17 Dic 2015.

CASTAÑO-URIBE, C., et al. 2015. Consideraciones particulares de los felinos en algunas zonas amortiguadoras de áreas protegidas del Caribe colombiano, Capítulo 11. Pp. 205. En: Payán, E., C. A. Lasso y C. Castaño-Uribe (Editores). 2015. I. Conservación de grandes vertebrados en áreas no protegidas de Colombia, Venezuela y Brasil. Serie Editorial Fauna Silvestre Neotropical. Instituto de Investigación de Recursos Biológicos Alexander von Humboldt (IAvH), Bogotá, D. C., Colombia.

CRAWSHAW JR., P.G. \& QUIGLEY, H.B. 1989. Notes on movement and activity of ocelots. Biotropica, 21: 301-307.

DI BITETTI, M., et al. 2005. Density Habitat Use and Activity Patterns of Ocelots (Leopardus Pardalis) in the Atlantic Forest of Misiones, Argentina. Journal of Zoology. 270: $153-163$.

DÍAZ - PULIDO A. \& PAYAN, E. 2011. Densidad De Tigrillos (Leopardus Pardalis) En Los Llanos Colombianos. Mastozoología Neotropical (18) 63 - 71.

DÍAZ - PULIDO A. \& PAYAN, E. 2012. Manual de Fototrampeo: Una Herramienta De Investigación Para La Conservación De La Biodiversidad En Colombia. Instituto De Investigaciones De Recursos Biológicos Alexander Von Humboldt y Panthera Colombia. 32 Pp.

DILLON, A. 2005. Ocelot Density and Home Range in Belize, Central America: Camera-Trapping and Radio Telemetry. Master Thesis. Virginia Polytechnic Institute and State University. $150 \mathrm{Pp}$.

EISENBERG, J.F. (Ed.) 1989. Mammals of the Neotropics. Vol. 1. The Northern Neotropics: Panama, Colombia, Venezuela, Guyana, Suriname and French Guiana. The University of Chicago Press. Chicago. 449p.

EMMONS. L.H.1988. A Field Study of Ocelots (Felis pardalis) In Peru. Rev. Ecol. (Terre Vie) 43: 133-157.

EMMONS, L.H. 1990. Neotropical Rainforest Mammals: A Field Guide. Chicago: The University of Chicago Press; 281 P.

GONZÁLEZ-MAYA, J., et al. 2013. Ecología y conservación de felinos y presas en el Caribe colombiano. Capítulo 6. En: Castaño-Uribe, C., González-Maya, J., Zárrate-Charry, D., Ange-Jaramillo, C. y Vela-Vargas, I. (Editores.) Plan de Conservación de Felinos del Caribe colombiano: Los felinos y su papel en la planificación regional integral basada en especies clave.

HAINES, A., et al. 2006. The importance of private lands for ocelot Leopardus pardalis conservation in the United Sates. Oryx Vol 40 No 1.

HOLDRIDGE, L. R. 1967. Life Zone Ecology. Tropical Science Center. San José, Costa Rica.

KARANTH, K.U. \& NICHOLS, J. D. 1998. Estimation of Tiger Densities in India Using Photographic Captures and Recaptures. Ecology 79(8), $2852-2862$.

KARANTH, K.U. \&. NICHOLS, J.D. 2002. Monitoring Tigers and Their Prey: A Manual for Researchers, Managers and Conservationists in Tropical Asia. Bangalore, Centre for Wildlife Studies.

KARANTH, K. U. 1995. Estimating Tiger Panthera Tigris Populations from Camera Traps Data Using Capture-Recapture Models. Biol.Conser. 71: 333 -336.

ROY, M., et al. 2015. Demystifying the Sundarban Tiger: Novel Application of Conventional Population Estimation Methods in a Unique Ecosystem. Popul. Ecol. Doi 10.1007/S10144-015-0527-9.

MINISTERIO DEL MEDIO AMBIENTE DE COLOMBIA 2000. Guía Para la Evaluación de Poblaciones de Fauna Silvestre. Bogotá 99 P.

MOREIRA, J., et al. 2007. Densidad de tigrillos (Carnívora: Leopardus Pardalis) en la parte Este del Parque Nacional Mirador Río Azul, Guatemala. 2007. Sociedad para la Conservación de la Vida Silvestre (WCS-Guatemala).

MORENO, R. \& BUSTAMANTE, A. 2007. Estatus del Jaguar, otros Felinos y sus Presas en el Alto Chagres, Utilizando Cámaras Trampa. Reporte Técnico. Sociedad Mastozoológica De Panamá. 28 P.

MORENO, R. \& GIACALONE, J. 2006. Ecological Data Obtained from Latrine use by Ocelots (Leopardus pardalis) on Barro Colorado Island, Panama. Tecnociencia vol. 8(1): $7-21$.

MORENO, R. 2000. Atrayentes para los Felinos Silvestres. Scientia 15 (1):115-117.

MORENO, R. 2002. Hábitos Alimentarios de Tigrillos (Leopardus Pardalis) y Pumas (Puma Concolor) en dos Localidades de la Cuenca del Canal de Panamá. Tesis de Licenciatura. Universidad de Panamá. 60 P.

MORENO, R. 2005. Nota: Observaciones sobre un Evento Antagónico en Tigrillos (Leopardus pardalis). Tecnociencia 7(2): $173-177$.

MORENO, R. et al. 2006.Competitive Release in the Diet of Ocelots (Leopardus pardalis) and Puma (Puma Concolor) after Jaguar (Panthera onca) Decline. J. Mammalogy 87 (4): 808-816.

MORENO, R. et al. 2006b. Un Estudio sobre la Ecología del tigrillo (Leopardus pardalis) en la isla de Barro Colorado, Panamá. Mesoamericana. X Congreso de la Sociedad Mesoamericana para la Biología y la Conservación, Ciudad Antigua, Guatemala.

MORENO, R. et al. 2012. Ámbito de Hogar y Actividad Circadiana del Tigrillo (Leopardus pardalis) en la isla de Barro Colorado, Panamá. Mesomamericana 16(3) 10 Pp. MINISTERIO DE AMBIENTE y DESARROLLO SOSTENIBLE DE COLOMBIA. 2017. Resolución 1912 de 15 de septiembre de 2017 . Por el cual se establece el listado de especies silvestres amenazadas de la diversidad biológica colombiana continental y marino costera que se encuentran en el territorio nacional y se dictan otras disposiciones. NAVARRO, D. 1985. Status and distribution of the ocelot (Felis pardalis) in South Texas. Tesis de Maestría. Texas University. Kingsville, EE. UU.

NOSS, A. et al. 2013. Evaluando la densidad de jaguares con trampas cámara. Programa para la Conservación del Jaguar Programa para Latinoamérica y el Caribe Wildlife Conservation Society Bronx, New York, 10460. 


\section{Artículo | Article}

OTIS, D.L., et al. 1978. Statistical inference from capture data on closed populations. Wildlife Monographs 62:1-135

PAYÁN, E. 2009. Hunting Sustainability, Species Richness and Carnivore Conservation in Colombian Amazonia. Tesis de Phd, Department of Biology and Department of Anthropology.University college London E Institute of Zoology. London.

REXSTAD, E., \& BURNHAM, K P. 1991. User's Guide for Interactive Program CAPTURE. Colorado Cooperative Fish \& Wildlife Research Unit, Colorado State University, Fort Collins, Colorado.

RUEDA-ALMONACID, J.V., et al. 2008. En: RODRÍGUEZ-MAHECHA, J.V., J. V. RUEDA-ALMONACID, T.D. GUTIÉRREZ H. (Editores.) 2008. Guía Ilustrada de la Fauna del Santuario de Vida Silvestre Los Besotes, Valledupar, Cesar, Colombia. Serie De Guías Tropicales De Campo N. ${ }^{\circ}$, Conservación Internacional-Colombia. Editorial Panamericana, Formas E Impresos. Bogotá, Colombia. 574 pp.

SEIDENSTICKER, J. \& LUMPKIN, S. 2004. Cats. Smithsonian Answer Book. J. Seidensticker \& S. Lumpkin; Photographs by Art Wolfe.254 Pp.

TORRES-ROMERO, E., et al. 2017. Ecology and conservation of ocelot (Leopardus pardalis) in Northern Quintana Roo, Mexico. THERYA, 2017 , Vol. 8 (1): 11 - 18 DOI: 10.12933/therya-17-439 ISSN 2007-3364.

TROLLE, M. \& KERY, M. 2003. Estimation of Ocelot Density in the Pantanal Using Capture-Recapture Analysis of Camera-Trapping Data. Journal of Mammalogy, 84(2): 607614.

TROLLE, M. \& KERY, M. 2005. Camera-Trap Study of Ocelot and other Secretive Mammals in the Northern Pantanal. Mammalia 69 (3-4): $405-412$. 